\title{
Contents, Vol. 57, 1925
}

\section{Inhalts-Verzeichnis.}

Über Blutaustritte in der Aderhaut. Von Prof. Dr. Ernst

Fuchs in Wien 1

Iritis serosa oder Cyclitis chronica? Von Prof. Dr. Ernst

Fuchs in Wien 6

Aus der Universitäts-Augenklinik in Innsbruck (Vorstand Prof. Dr. R. Seefeläer).

Zur Operation des komplizierten Stares. Von Prof. Dr.

R. Seefelder 12,91

Über Wundsprengungen nach Staroperationen. Von Dr. H.

Gasteiger 21

Über zwei Fälle von primären intraduralen Sehnerventumoren.

Von Dr. Ko Hi dan o (in Tokio) 31

Beiträge zur sympathischen Ophthalmic Von Dr. O. March esani. (Hierzu Tafel I-IV.) 44

Über gehäu†tes Auftreten von Neuritis retrobulbaris und der enBehandlung mit der

„Daueranämisierungu. VonProf. Dr. F. v. Herrenschwand 78

Aus der Universitäts-Augenklinik in Graz. (Vorstand : Prof. Dr. Salzmann).

Über eine Abart der knötchenförmigen Hornhautdystrophie.

Von Prof. Dr. Maximilian Salzmann. (Hierzu

Tafel V.) 92

Markhaltige Nervenfasern und Sehnervenentzündung. Von

Prof. Dr. Robert Hesse. (Hierzu Tafel VI.) . . 100 Über Atrophia nervi optici partialis nach

schweren Blutungen.

Von Dr. Walter Grimminger 106

Die Preglsche Jodlösung in der Augenheilkunde. Von Dr.

Rudolf Schneider $\quad 121$

Aus der I. Universitäts-Augenklinik in Wien.

Über spontane Blutungen in atrophischen Augen. Von Prof.

Dr. J. Me Her. (Hierzu Tafel VII/VIII.) . . . 131 Über ein Karzinom der Conjunctiva tarsi. Von

Dozenten

Dr. Ernst Bachstez 153

Zur Kenntnis der gittrigen Hornhautentartung (Haab-Dimmer).

Von Dozenten Dr. Adalbert Fuchs. (Hierzu

Tafel IX.) 159

$-\mathrm{VI}-$

Über eine Orbitalcyste an Stelle des mißbiläeten Optikus.

(Ein Beitrag zur Klinik und Genese der Orbital-

zysten.) Von Dr. Karl Safar 186

Eine Methode der West-Polyakschen endonasalen Tränensack-

operation. Von Priv.-Doz. Dr. Karl Kofler und 
Dr. Josef Urbanek 200

Zur Frage des ursächlichen Zusammenhanges zwischen Trauma

und Chorioidealsarkom. Von Dr. Maximilian

Nitsch 225

Über idiopathischen Zerfall des retinalen Pigmentblattes der

Iris bei zwei Brüdern. Von Dr. H. Mauksch.

(Hierzu Tafel X.) .... 262

Über eine sehr seltene kongenitale Pigmentblatíanomalie des

Ziliarkörpers. Von Dr. Peter KronfeId . . . 269 Über Runãzellenherde in der Uvea atrophischer

Augen. Von

Dr. Stephan Zimmermann 279

Zur Kenntnis der perivaskulären Tuberkuloseverbreitrung im

Auge. Von Dr. Stephan Frank301

Iris bombé mit und ohne Drucksteigerung. Von Dr. E11 o r e

D’Oswaldo in Görz. (Hierzu Tafel XI.) . . . 311

Aus der zweiten Universitäts-Augenklinik in Wien. (Vorstand : Prof. Dr. Dimmer).

Über den Einfluß der Rech ¿s- und Linkshändigkeit auf dieEntwicklung des führenden Auges und des Strabismusconcomitansunilateralis. VonDoz.Dr.Rich.Kramerund Dr. Melan. Schützenhuber 322

Über xenogene Siäerosis bulbi. Von Dr. Gustav Guist.

(Hierzu Tafel XII und XIII.) 335

Über hintere Glaskörperabhebung. Von Dr. Arnold Pillat.

(Hierzu Tafel XIV.) . 347

Über Hornhautverkupferung nach therapeutischer Anwen-dung von Kupferpräparaten. Von Dr.

LudwigSallmann. (Hierzu Tafel XV.) 373

Über die Netzhautveränderungen bei Nieren- und Gefäßer-krankungen. (Zugleich ein Beitrag zur

Pathogeneseder Retinitis nephritica). Von Dr. H. Kahler undDr. L. Sallmann 386

Ein weiterer Fall von ,,präretinalem Ödem”. Von Dr.

Charles J. Gissy aus St. Louis 423

Ein Beitrag zur Kasuistik der tapeto-retinalen Degeneration.(Heredodegeneration der Makula mit Beteiligungder Peripherie bei drei Brüdern). Nebst Bemer-kungen über den Erbgang der

Heredodegenerationder Makula. Von Dr. Herwigh Rieger. (Hierzu Tafel XVI-XVII.)

429

- VII -

Aus der Augenabteilung des Wiedner Krankenhauses Wien (Vorstand Prof, Dr. M. Sachs).

Notizen zur Lehre von den phantastischen Gesichtserschei-

nungen. Von Prof. Dr. M. Sachs 464

Über eine Vorrichtung zur kontinuierlichen Bestimmungder Seh-

schärje. Von Dr. Marie Proksch 472

Aus der Augenabteilung des Lainzer Jubiläumsspital der Gemeinde Wien (Vorstand Prof. Dr. H.

Laiǿer).

Ein Normalperimefer als Grundlage der Vereinheitlichung

der Gesichtsfeldaufnahmen. Von Prof. Dr. H. L a u b e r 481

Ein Fall von Hemiatro $+>$ hia facialis progr'essiva mit Betei-ligung des Auges. Von Prof. Dr. H.

Lauber.(Hierzu Tafel XVIII-XX.) 492

Aus dem Rudolfspital Wien (Vorstand Prof. Dr. Hanke). 
Das Trachom im Burgenlande und seine Bekämpfung. Von

Prof. Dr. V. Hanke 497

Aus der Augenabteilung der Wiener Allgemeinen Poliklinik (Vorstand Prof. Dr. K. Lindner).

Über die Schwierigkeiten der Trachomforschung. Von Prof.

Dr. K. Lindner. (Hierzu Tafel XXI) 508

Zum Mechanismus der Fixation. (Eine experimentelle

Studie). Von Dr. A. Kestenbaum 557

Aus der Laryngol. Abteilung des Kaiser Franz Joseph-Ambulatoriums und Jubiläumsspitals in Wien (Vorstand : Priv.-Doz. Dr. Oskar Hirsch).

Die Wide? stands jähigkeit der zentralen Sehbahnen gegenübermechanischem Druck. Von

Priv.-Doz. Dr. OskarHirsch in Wien 592

Aus der Augenabteilung des Landeskrankenhauses in Klagenfurt (Primarius Dr. Adolf

Purtscher).

Ent†emung einer lebenden Larve von Hypoderma bovis aus

dem Glaskörper. Von Dr. Adolf Purtscher . . 601

Aus der Universitäts-Augenklinik der k. Universität in Zagreb (Vorstand Prof. Dr. A. Botteri).

Anatomischer Beitrag zum intraokularen Zystizerkus. Von

Dr. Vasilj Derkaõ 605

Schwâche der glatten Irismuskulatur beim Diabetes. Von

Dr. A. Car 614

Mikrozephalie und beiderseitiges Kolobom im Bereiche der

Makula. Von Dr. A. Car 618

Ein bemerkenswerter Fall von Feuermal und Glaukom. Von

Primärarzt Dr. Carl Bar in Meran $\quad 628$

S. Sachverzeichnis Namenverzeichnis

631632 